\title{
Syndrome de Klinefelter et conseil génétique
}

\author{
J. P. SIFFROI
}

Service d'Histologie, Biologie de la Reproduction et Cytogénétique

Hôpital Tenon, 4 rue de la Chine, 75020 Paris

RÉSUMÉ

Le syndrome de Klinefelter a longtemps été considéré comme synonyme de stérilité masculine jusqu'à l'apparition des techniques d'ICSI. La possibilité pour des hommes à caryotype anormal d'avoir une descendance propre a, dès lors, posé la question du risque génétique pour cette dernière. Grâce aux méthodes d'analyse des chromosomes des spermatozoïdes, après fécondation hétérospécifique homme-hamster ou par hybridation in situ de sondes fluorescentes, il a pu être montré que les spermatozoïdes retrouvés chez un nombre non négligeable de sujets Klinefelter avaient le plus souvent un contenu chromosomique équilibré, ce qui a été confirmé par la normalité du caryotype de la plupart des enfants nés après ICSI à partir de ces hommes. Cependant, l'augmentation, faible mais significative chez eux, des spermatozoïdes disomiques pour les gonosomes laisse penser que leur spermatogenèse s'effectue dans un environnement testiculaire défavorable quant au contrôle de la ségrégation chromosomique à la méiose. Cette constatation peut être étendue aux autosomes et incite à la prudence dans le suivi génétique des grossesses.

Mots clés : Syndrome de Klinefelter, FISH, ICSI, Conseil génétique.

\section{INTRODUCTION}

Parler de conseil génétique dans le syndrome de Klinefelter peut paraître paradoxal. En effet, par définition, une consultation de conseil génétique a pour but de définir les causes génétiques d'une pathologie, d'en préciser le caractère sporadique ou familial et le mode de transmission dans une famille donnée et surtout, ce qui est primordial pour les patients, de prévoir le risque pour la descendance. Les éventuelles possibilités de diagnostic prénatal visant à couvrir ce risque font également partie du conseil génétique. Or, le syndrome de Klinefelter étant classiquement associé à une stérilité, le risque pour la descendance n'existait pas jusqu'à ce que les nouvelles techniques d'aide médicale à la procréation par micro-injection intracytoplasmique de spermatozoïde (ICSI) ne viennent bouleverser cette situation en offrant la possibilité à certains sujets atteints d'avoir des enfants. Curieusement, dans ces cas, la question du risque génétique ne se limite pas forcément à la transmission de l'anomalie parentale mais peut être d'ordre plus général.

Correspondance : J. P. Siffroi, Service d'Histologie, Biologie de la Reproduction et Cytogénétique, Hôpital Tenon, 4 rue de la Chine, 75020 Paris.

e-mail : jean-pierre.siffroi@tnn.ap-hop-paris.fr

Communication au XVIIème Congrès de la SALF, 7-8 décembre 2000, Bordeaux 


\section{DES RELATIONS GÉNOTYPE- PHÉNOTYPE DIFFICILES À ÉTABLIR}

Le diagnostic formel de syndrome de Klinefelter est posé devant la constatation d'une formule chromosomique de type $47, \mathrm{XXY}$ dont le caractère homogène ou en mosaïque ne peut être établi avec certitude. En effet, en pratique courante, le caryotype est réalisé par l'analyse d'une vingtaine de lymphocytes circulants et la normalité de ce dernier sur ces cellules ne préjuge pas de l'existence éventuelle dans l'organisme, et notamment au niveau testiculaire, de cellules possédant une formule caryotypique anormale de type 47, XXY. A l'inverse, la constatation d'un caryotype anormal et homogène dans le sang ne signifie pas forcément l'absence de cellules $46, \mathrm{XY}$ en proportions variables dans d'autres tissus. Partant de l'observation qu'au moins $10 \%$ des cas de syndrome de Klinefelter présentent une mosaïque détectable d'emblée dans les cellules sanguines, il est très probable qu'un pourcentage encore plus important d'entre eux soit porteur d'une mosaïque cryptique expliquant soit la persistance d'une spermatogenèse chez des sujets apparemment homogènes $47, \mathrm{XXY}$ soit une atteinte de cette dernière chez d'autres avec un caryotype normal. Cette situation fait que certains hommes atteints d'un syndrome de Klinefelter se présentent non pas avec une azoospermie mais avec une oligozoospermie, le plus souvent extrême, et que la question de l'opportunité d'une biopsie testiculaire se pose chez ceux qui sont azoospermiques mais avec des paramètres cliniques et biologiques qui laissent espérer la conservation d'une certaine spermatogenèse [24]. Le conseil génétique reprend alors toute sa valeur dans l'estimation du risque pour la descendance.

\section{LES MOYENS D'ÉTUDE DE LA " QUALITÉ " CHROMOSOMIQUE DES SPERMATOZOÏDES}

Outre le fait d'être à l'état haploïde et dans un noyau de type interphasique, le génome des spermatozoïdes est caractérisé par un niveau de condensation très important qui empêche son analyse par les techniques de cytogénétique classiques. Deux méthodes sont cependant disponibles qui permettent l'étude des chromosomes contenus dans le noyau de ces cellules. La première consiste à réaliser un test de fécondation hétéro-spécifique homme-hamster, à obtenir la décondensation des têtes spermatiques dans l'ovocyte et, après culture, à visualiser un ou plusieurs lots haploïdes de chromosomes humains dont l'aspect va être très proche de celui des chromosomes mitotiques classiques. Cette technique a l'avantage de fournir des renseignements sur la totalité $\mathrm{du}$ caryotype, anomalies de structure comprises, mais elle présente l'inconvénient d'être difficile à maîtriser et de ne permettre l'analyse que d'un nombre limité de spermatozoïdes par patient. La seconde est représentée par l'hybridation in situ en fluorescence (FISH) de sondes marquées, spécifiques de tel ou tel chromosome, sur des lames de frottis de spermatozoïdes dont les têtes ont été préalablement décondensées. Cette méthode permet d'analyser rapidement un grand nombre de cellules, jusqu'à 10000 par patient, mais ne donne des informations que sur les anomalies de nombre pour les chromosomes testés. En pratique, dans le syndrome de Klinefelter, les sondes utilisées sont des sondes centromériques codant pour les chromosomes $\mathrm{X}$ ou $\mathrm{Y}$ auxquelles est rajoutée une sonde codant pour un autosome de façon à mesurer la ploïdie des cellules et à différencier des spermatozoïdes disomiques 24, XY de cellules diploïdes, 46, XY.

Ces deux techniques de laboratoire ont été largement utilisées pour établir le taux de base des aneuploïdies dans les spermatozoïdes humains et de nombreuses études en font état à la fois chez des sujets normaux, que ce soit par rapport à leur sperme ou à leur caryotype, mais aussi chez des patients infertiles à caryotype normal [25]. Elles ont également permis d'étudier le mode de ségrégation de certaines anomalies caryotypiques équilibrées, comme des translocations ou des inversions [9]. Enfin, de nombreuses corrélations ont pu être faites entre la fréquence des anomalies chromosomiques dans le sperme et certains paramètres comme l'âge [22] ou les techniques de préparation des spermatozoïdes comme la cryopréservation [3]. 


\section{DISCORDANCE ENTRE LES MODES DE SÉGRÉGATION THÉO- RIQUES ET OBSERVÉS DANS LES SPERMATOZOÏDES DES SUJETS PRESENTANT UN SYNDROME DE KLINEFELTER}

Les études faisant état de la fréquence des aneuploïdies, notamment celles touchant les gonosomes, dans les spermatozoïdes des hommes $47, \mathrm{XXY}$, homogènes ou le plus souvent en mosaïque, sont rares et ne représentent que des " case reports " dans la littérature $[4,5,6,7,8,10,12,14,16,20]$. Néanmoins, ces publications s'accordent sur le fait qu'il existe bien une augmentation des formes disomiques 24, XX ou 24, XY dans les spermatozoïdes de ces sujets mais dans une proportion très largement inférieure à ce que ne le voudrait un schéma classique de ségrégation des gonosomes à partir d'une spermatogonie $47, \mathrm{XXY}$ qui arriverait à achever sa méïose. Dans un tel schéma en effet, les pourcentages des formes équilibrées et disomiques seraient soit identiques (25\%), en cas de ségrégation totalement aléatoire des gonosomes, soit décalés, avec une majorité de formes normales $23, \mathrm{X}$ et une augmentation relative des formes $24, \mathrm{XY}$, en cas d'appariement préférentiel des deux chromosomes $\mathrm{X}$ au stade pachytène de la méïose.

En fait, que ce soit chez les sujets présentant un syndrome de Klinefelter apparemment homogènes ou en mosaïque, le taux de spermatozoïdes disomiques pour les gonosomes est compris entre $1 \%$ et $2 \%$, avec une augmentation légèrement plus forte pour les formes $24, \mathrm{XY}$ par rapport à celles $24, \mathrm{XX}[1,16,4]$, en dehors de quelques publications qui font état de chiffres plus élevés [7] mais toujours à partir d'un nombre très réduit de patients. De plus, certaines études montrent également une élévation faible mais significative des formes 24, YY qui traduisent une erreur de ségrégation en deuxième division méiotique, sans rapport apparent avec un schéma de ségrégation théorique en première division de méiose à partir d'une cellule 47, XXY [1,16]. Enfin, les techniques de FISH sur spermatozoïdes incluant systématiquement une sonde codant pour un autosome, certains auteurs notent aussi une augmentation des disomies pour le chromosome testé ce qui, là encore, ne présente pas de rapport direct avec les anomalies de ségrégation des gonosomes [20].

Dans la plupart des études, le taux d'aneuploïdie dans le sperme des sujets présentant un syndrome de Klinefelter est comparé avec celui existant chez des sujets contrôles fertiles ou normospermiques et la différence est alors le plus souvent considérée comme significative. Cependant, il est connu que des hommes infertiles à caryotype apparemment normal $46, \mathrm{XY}$ présentent également des fréquences augmentées de spermatozoïdes chromosomiquement anormaux, identiques à celles des patients présentant un syndrome de Klinefelter, ce qui pourrait être une explication à l'élévation des anomalies gonosomiques chez les enfants conçus par ICSI $[8,15,20]$. Le problème ne serait donc plus le risque génétique lié à la présence de cellules testiculaires $47, \mathrm{XXY}$ mais plutôt celui du contrôle général de la ségrégation chromosomique au cours d'une spermatogenèse de mauvaise qualité.

\section{DES CELLULES À CARYOTYPE 46, XY EFFECTUANT LEUR MÉÏOSE DANS UN " ENVIRONNEMENT" TESTICULAIRE DÉFAVORABLE}

Il est donc probable que seules des cellules 46 , $\mathrm{XY}$, présentes chez les sujets présentant un syndrome de Klinefelter en mosaïque, au moins au niveau testiculaire, sont capables d'effectuer une spermatogenèse complète mais que cette dernière, et notamment la phase de mêiose, se déroulerait dans un environnement testiculaire défavorable, c'est à dire sous le contrôle endocrine et paracrine de cellules à caryotype anormal, 47, XXY. Cette spermatogenèse, quantitativement faible, serait alors aussi de qualité médiocre ce qui se traduirait par une élévation des erreurs de ségrégation méiotique, à la fois en première mais aussi en seconde division de méiose, expliquant ainsi l'augmentation relative des spermatozoïdes disomiques pour l'un ou l'autre des gonosomes ou encore de ceux présentant une anomalie de nombre pour un autosome.

Un tel modèle est appuyé par des observations 
faites chez des souris mâles XXY dont le phénotype testiculaire rappelle celui des hommes présentant un syndrome de Klinefelter. Ces études ont tout d'abord montré que les spermatogonies de ces souris, qu'elles soient prépubères ou adultes, avaient toujours un caryotype XY et que les rares spermatozoïdes produits provenaient de segments ponctuels des tubes séminifêres, indiquant par là leur formation à partir de clones cellulaires ayant perdu le chromosome $\mathrm{X}$ surnuméraire [17]. Elles ont également retrouvé une augmentation faible mais significative des disomies gonosomiques, tout comme celle observée chez les patients présentant un syndrome de Klinefelter.

Chez l'homme, une étude en FISH réalisée sur des biopsies testiculaires pratiquées chez des patients à caryotype $47, \mathrm{XXY}$, homogènes ou non, a permis de constater que certaines cellules pré-méiotiques ( $80 \%$ chez un patient homogène contre $25 \%$ chez deux en mosaïque) pouvaient présenter un caryotype anormal $\mathrm{XXY}$ mais que tous les spermatocytes au stade pachytène étaient de type $\mathrm{XY}$, traduisant ainsi le fait que seules des cellules à caryotype normal étaient capables d'entrer en méíose [2]. Là encore, la constatation de cellules post-méïotiques disomiques pour l'X ou l'Y en nombre relativement élevé ( $18 \%$ et $2 \%$ ) indique que la spermatogenèse de ces sujets se déroule dans un environnement délétère quant au contrôle de la ségrégation chromosomique.

\section{UN CONSEIL GÉNÉTIQUE PLUTÔT RASSURANT}

Il n'existe pas encore de recul suffisant ni d'étude à grande échelle pour pouvoir affirmer que des grossesses obtenues par ICSI à partir d'hommes Klinefelter présentent un faible risque génétique pour la descendance. Cependant, à notre connaissance, un seul cas de transmission père-fils d'un caryotype 47 , XXY a été décrit dans la littérature [21] et les quelques données tirées du diagnostic prénatal [11, 13, 18, 19], voire pré-implantatoire [23], sont rassurantes. La question la plus critique concerne en fait le risque d'augmentation des aneuploïdies touchant les autosomes dans ces grossesses, risque qu'il est encore impossible de chiffrer mais qui est peut-être réel si on admet que la spermatogenèse des sujets présentant un syndrome de Klinefelter s'accompagne d'un mauvais contrôle de la ségrégation des chromosomes en général. Dès lors, il est licite de proposer d'une part une analyse en FISH des spermatozoïdes d'un sujet présentant un syndrome de Klinefelter candidat à l'ICSI, pour mesurer de façon individuelle le taux d'anomalies chromosomiques dans ses spermatozoïdes, et d'autre part un diagnostic prénatal en cas de grossesse évolutive tout en prévenant les couples de la situation difficile qui pourrait résulter de la mise en évidence chez un foetus de la même anomalie chromosomique que celle affectant son père.

\section{RÉFÉRENCES}

1. BIELANSKA M., TAN S.L., AO A. : Fluorescence in situ hybridization of sex chromosomes in spermatozoa and spare preimplantation embryos of a Klinefelter syndrome 46,XY / 47, XXY male. Hum. Reprod., 2000, $15: 440-444$.

2. BLANCO J., EGOZCUE J., VIDAL F. : Apoptosis and non-disjunction in XXY and XYY. Hum. Reprod., 2000, 15, abst.1, 48 .

3. CHERNOS J.E., MARTIN R.H. : A cytogenetic investigation of the effects of cryopreservation on human sperm. Am. J. Hum. Genet., 1989, 45 : 766-777.

4. CHEVRET E., ROUSSEAUX S., MONTEIL M. et al. : Increased incidence of hyperhaploid $24, \mathrm{XY}$ spermatozoa detected by three-colour FISH in a $46, \mathrm{XY}$ / 47,XXY male. Hum. Genet., 1996, 97 : 171-175.

5. COZZI J., CHEVRET E., ROUSSEAUX S.,et al. : Achievement of meiosis in XXY germ cells : study of 543 sperm karyotypes from an XY / XXY mosaic patient. Hum. Genet., 1994, 93 : 32-34.

6. ESTOP A.M., MUNNE S., CIEPLY K.M., VANDERMARK K.K., LAMB A.N., FISCH H. : Meiotic products of a Klinefelter 47 , XXY male as determined by sperm fluorescence in situ hybridization analysis. Hum. Reprod., 1998, 13 : 124-127.

7. FORESTA C., GALEAZZI C., BETTELLA A, et al. : Analysis of meiosis in intratesticular germ cells from subjects affected by classic Klinefelter's syndrome. J. Clin. Endocrinol. Metab., 1999, 84 : 3807-3810.

8. GILTAY J.C., VAN GOLDE R.J.T., KASTROP P.M.M. : Analysis of spermatozoa from seven ICSI males with constitutional sex chromosomal abnormalities by fluorescent in situ hybridization. J. Assist. Reprod. Genet., 2000, $17:$ 151-155.

9. GUTTENBACH M., ENGEL W., SCHMID M. : Analysis of structural and numerical chromosome abnormalities in sperm of normal men and carriers of constitutional chromosome aberrations. A review. 
Hum. Genet., 1997, $100:$ 1-21.

10. GUTTENBACH M., MICHELMANN H.W., HINNEY B., ENGEL W., SCHMID M. : Segregation of sex chromosomes into sperm nuclei in a man with $47, \mathrm{XXY}$ Klinefelter's karyotype : a FISH analysis. Hum. Genet., 1997, $99: 474-477$.

11. HINNEY B., GUTTENBACH M., SCHMID M., ENGEL W., MICHELMANN H.W. : Pregnancy after intracytoplasmic sperm injection with sperm from a man with a 47 , XXY Klinefelter's karyotype. Fertil. Steril., 1997, $68:$ 718-720.

12. KRUSE R., GUTTENBACH M., SCHARTMANN B. $e t$ al. : Genetic counselling in a patient with XXY / XXXY / XY mosaic Klinefelter's syndrome : estimate of sex chromosome aberrations in sperm before intracytoplasmic sperm injection. Fertil. Steril., 1998, 69 : 482485.

13. LEVRON J., AVIRAM-GOLDRING A., MADGAR I., RAVIV G., BARKAI G., DOR J. : Sperm chromosome analysis and outcome of IVF in patients with nonmosaic Klinefelter's syndrome. Fertil. Steril., 2000, 74 : 925-929.

14. LIM A.S., FONG Y., YU S.L. : Estimates of sperm sex chromosome disomy and diploidy rates in a $47, \mathrm{XXY} /$ 46,XY mosaic Klinefelter patient. Hum. Genet., 1999, $104: 405-409$.

15. MOOSANI N., CHERNOS J., LOWRY R.B., MARTIN R.H. : A 47, XXY fetus resulting from ICSI in a man with an elevated frequency of 24 , XY spermatozoa. Hum. Reprod., 1999, 14 : 1137-1139.

16. MOREL F., ROUX C., BRESSON J.L. : Segregation of sex chromosomes in spermatozoa of $46, \mathrm{XY} / 47, \mathrm{XXY}$ men by multicolour fluorescence in situ hybridization. Mol. Hum. Reprod., 2000, 6 : 566-570.

17. MROZ K., HASSOLD T.J., HUNT P.A. : Meiotic aneuploidy in the XXY mouse : evidence that a compromised testicular environment increases the incidence of meiotic errors. Hum. Reprod., 1998, 14 :1151-1156.

18. NODAR F., DE VINCENTIIS S., OLMEDO S.B., PAPIER S., URRUTIA F., ACOSTA A.A. : Birth of twin males with normal karyotype after intracytoplasmic sperm injection with use of testicular spermatozoa from a nonmosaic patient with Klinefelter's syndrome. Fertil. Steril., 1999, 71 : 1149-1152.

19. REUBINOFF B.E., ABELIOVICH D.A., WERNER M., SCHENKER J.G., SAFRAN A., LEWIN A. : A birth in non-mosaic Klinefelter's syndrome after fine needle aspiration, intracytoplasmic sperm injection and preimplantation diagnosis. Hum. Reprod., 1998, $13: 1887-1892$.

20. RIVES N., JOLY G., MACHY A., SIMEON N., LECLERS P., MACE B. : Assessment of sex chromosome aneuploidy in sperm nuclei from $47, \mathrm{XXY}$ and 46, XY / 47, XXY males : comparison with fertile and infertile males with normal karyotype. Mol. Hum. Reprod., 2000, 6 : 107-112.
21. RON-EL R., STRASSBURGER D., GELMANKOHAN S., FRIEDLER S., RAZIEL A., APPELMAN Z. : A 47, XXY fetus conceived after ICSI of spermatozoa from a patient with non-mosaic Klinefelter's syndrome : case report. Hum. Reprod., 2000, 15 : 18041806.

22. ROUSSEAUX S., HAZZOURI M., PELLETIER R., MONTEIL M., USSON Y., SELE B. : Disomy rates for chromosomes 14 and 21 studied by fluorescent in-situ hybridization in spermatozoa from three men over 60 years of age. Mol. Hum. Reprod., 1998, 4 : 695-699.

23. STAESSEN C., COONEN E., VAN ASSCHE E. et al.: Preimplantation diagnosis for $\mathrm{X}$ and $\mathrm{Y}$ normality in embryos from three Klinefelter patients. Hum. Reprod., 1996, 11 : 1650-1653.

24. TOURNAYE H., STAESSEN C., LIEBAERS I. et al. : Testicular sperm recovery in nine 47, XXY Klinefelter patients. Hum. Reprod., 1996, 11 : 1644-1649.

25. VEGETTI W., VAN ASSCHE E., FRIAS A. et al. : Correlation between semen parameters and sperm aneuploidy rates investigated by fluorescence in-situ hybridization in infertile men. Hum. Reprod., 2000, $15: 351-365$.

\author{
ABSTRACT \\ Genetic counselling in Klinefelter's \\ syndrome
}

J.P. SIFFROI

Klinefelter's syndrome is a common sex chromosomal aberration generally characterized by hypergonadotrophic hypogonadism and azoospermia. However, spermatogenesis impairment is variable and severe oligozoospermia can be found in some men, particularly those exhibiting a mosaic karyotype 47,XXY / 46,XY. New reproductive technologies, such as intracytoplasmic sperm injection (ICSI), allow Klinefelter patients to have a progeny, even those who are azoospermic after testicular sperm recovery. The question therefore arises of whether or not there is a genetic risk for pregnancies from affected fathers. Sperm karyotyping, by in vitro penetration of zona-free hamster eggs or by fluorescence in-situ hybridization (FISH), is a 
method of choice for measuring aneuploidy rate in spermatozoa of patients carrying gonosomal abnormalities. A theoretical model would predict a high level of $24, \mathrm{XX}$ and/or 24,XY disomic sperm cells in Klinefelter patients if $47, \mathrm{XXY}$ spermatogonia were able to complete meiosis and achieve spermatogenesis. Interestingly, current observations show that the rate of abnormal spermatozoa in these patients is low, around 1-2\%, which indicates that only $46, \mathrm{XY}$ spermatogonia can produce mature sperm cells and that oligozoospermic Klinefelter patients probably carry a 47,XXY / 46,XY mosaicism, at least at the testicular level. However, this low but statistically significant level of disomic spermatozoa emphasizes the fact that their spermatogenesis occurs in a compromised environment which could increase the risk of meiotic errors. Therefore, the possible occurrence of autosomal aneuploidies in children born from Klinefelter fathers leads to the following recommendations: a) individual analysis by FISH of the sperm aneuploidy rate in each Klinefelter patient candidate for ICSI; b) proposal of fetal karyotyping after amniocentesis in pregnancies obtained by this technique.

Key Words: Klinefelter's syndrome, FISH, ICSI, genetic counselling. 